\title{
The Relation of Brain Oscillations to Attentional Networks
}

\author{
Jin Fan, ${ }^{1,2}$ Jennie Byrne, ${ }^{1}$ Michael S. Worden, ${ }^{3}$ Kevin G. Guise, ${ }^{1}$ Bruce D. McCandliss, ${ }^{4}$ John Fossella, ${ }^{1}$ and \\ Michael I. Posner ${ }^{4,5}$ \\ ${ }^{1}$ Departments of Psychiatry and ${ }^{2}$ Neuroscience, Mount Sinai School of Medicine, New York, New York 10029, ${ }^{3}$ Brain Science Program, Brown University, \\ Providence, Rhode Island 02912, ${ }^{4}$ Sackler Institute for Developmental Psychobiology, Weill Medical College of Cornell University, New York, New York \\ 10021, and ${ }^{5}$ Department of Psychology, University of Oregon, Eugene, Oregon 97403
}

Previous studies have suggested the relation of particular frequency bands such as theta $(4-8 \mathrm{~Hz})$, alpha $(8-14 \mathrm{~Hz})$, beta (14-30 $\mathrm{Hz})$, or gamma $(>30 \mathrm{~Hz})$ to cognitive functions. However, there has been controversy over which bands are specifically related to attention. We used the attention network test to separate three anatomically defined brain networks that carry out the functions of alerting, orienting, and executive control of attention. High-density scalp electrical recording was performed to record synchronous oscillatory activity and power spectrum analyses based on functional magnetic resonance imaging constrained dipole modeling were conducted for each attentional network. We found that each attentional network has a distinct set of oscillations related to its activity. The alerting network showed a specific decrease in theta-, alpha-, and beta-band activity $200-450 \mathrm{~ms}$ after a warning signal. The orienting network showed an increase in gamma-band activity at $\sim 200 \mathrm{~ms}$ after a spatial cue, indicating the location of a target. The executive control network revealed a complex pattern when a target was surrounded with incongruent flankers compared with congruent flankers. There was an early $(<400$ $\mathrm{ms})$ increase in gamma-band activity, a later $(>400 \mathrm{~ms})$ decrease in beta- and low gamma-band activity after the target onset, and a decrease of all frequency bands before response followed by an increase after the response. These data demonstrate that attention is not related to any single frequency band but that each network has a distinct oscillatory activity and time course.

Key words: attention; attentional networks; ERP; fMRI; source analysis; time course; oscillations

\section{Introduction}

There has been a great deal of interest in frequency-specific oscillatory activity in relation to cognitive functions (Kahana et al., 2001; Herrmann et al., 2004; Kahana, 2006). For example, the suppression of scalp-recorded alpha has been associated with general alerting when people are given a cue as to when a target will occur (Babiloni et al., 2004) and with changed activity within the visual cortex when attending to a target (Yamagishi et al., 2005). During attention to a visual target, increases in alpha activity at unattended locations have been related to the success of distracter suppression (Worden et al., 2000). Increased gammaband activity has been associated with visual attention in scalp recordings (Keil et al., 2001) and in studies of neural activity within the visual system (Fries et al., 2001; Womelsdorf et al., 2006). Gamma-band activity has also been associated with awareness of stimuli, another important aspect of attention (Crick and Koch, 1990). Theta activity has been associated with aspects of task monitoring, including error detection, that are often associated with executive attention (Luu et al., 2004). Beta-band activity has been found to synchronize between two remote electrode sites at the time information is being passed from one brain area

\footnotetext{
Received Aug. 16, 2006; accepted May 4, 2007.

This work was supported by National Science Foundation Grant BCS 9907831 and by a DeWitt Wallace-Reader's Digest Research Fellowship in Psychiatry.

Correspondence should be addressed to Dr. Jin Fan, Laboratory of Neuroimaging, Department of Psychiatry, Mount Sinai School of Medicine, One Gustave L. Levy Place, P.0. Box 1230, New York, NY 10029. E-mail: Jin.Fan@mssm.edu.

DOI:10.1523/JNEUROSCI.1833-07.2007

Copyright $\odot 2007$ Society for Neuroscience $\quad$ 0270-6474/07/276197-10\$15.00/0
}

to another (Nikolaev et al., 2001). This transfer of information between remote locations could be considered another function of attention.

Because attention is not a unitary function, it is important to relate these oscillations to different attentional networks. Human attentional processes can be conceptualized as a system of anatomical areas consisting of three specialized networks (Posner and Petersen, 1990). These networks carry out the functions of alerting to achieve and maintain an alert state, orienting to turn attention toward a sensory signal, and executive control to monitor and resolve conflict in the presence of competing information. We developed the attention network test (ANT), which provides measures of the function of these three attentional networks. We have shown that these networks are roughly independent in a behavioral study (Fan et al., 2002) and that they involve different anatomical areas as revealed by a functional magnetic resonance imaging (fMRI) study (Fan et al., 2005). The alerting network showed strong thalamic activation and activation of anterior and posterior cortical sites, the orienting network showed activation of expected frontal and parietal sites, and the executive control network showed activation of the anterior cingulate cortex (ACC) along with several other brain areas, such as the lateral prefrontal cortex.

In this study, we investigated whether these three networks are related to the same or different oscillatory neuronal dynamics. To achieve this, we conducted a high-density event-related potential (ERP) study using the ANT on participants, some of whom previously participated in the fMRI study (Fan et al., 2005). Dipole source modeling was performed using spatial constraints pro- 


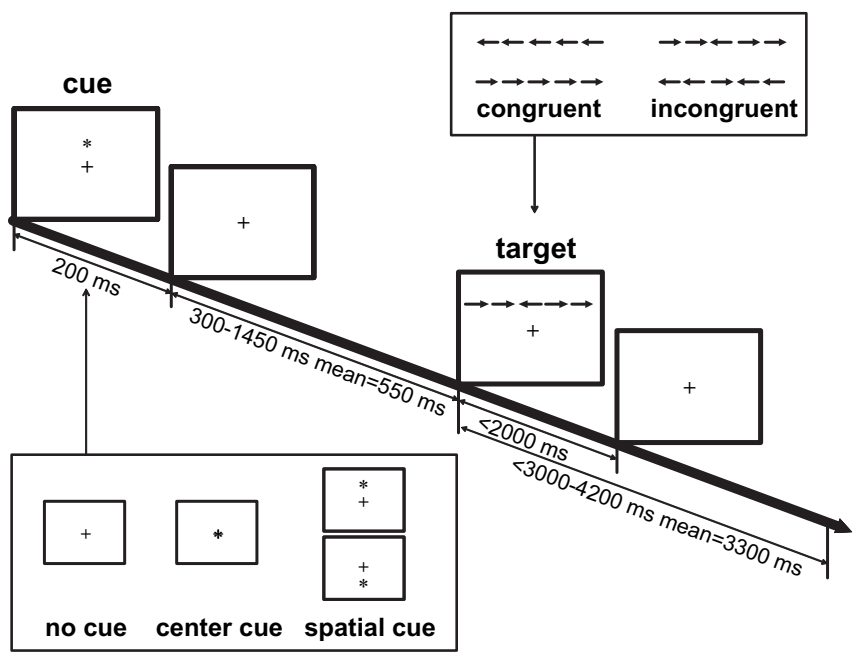

Figure 1. Schematic of the ANT. A fixation cross appears in the center of the screen all of the time. In each trial, depending on the cue condition (no cue, center cue, or spatial cue), a cue may appear for $200 \mathrm{~ms}$. After a variable duration ( $300-1450 \mathrm{~ms})$, the target (the center arrow) and flankers of two left and two right arrows (congruent or incongruent flankers) are presented. The participant makes a response to the target's direction within a time window of $2000 \mathrm{~ms}$. The target and flankers disappear after the response is made. The target and post-target fixation period lasts for a variable duration ( $3000-4200 \mathrm{~ms})$.

vided by the activation from the fMRI study and power spectrum analysis was conducted to examine synchronous activity change associated with the processes of these attentional networks.

\section{Materials and Methods}

Participants. Thirty-six right-handed neurologically normal adults (mean age, 27.2 years; SD, 5.2, range, 19-36 years; 18 male), 12 of whom participated in the event-related fMRI study (Fan et al., 2005), participated this study. Participants had normal or corrected to normal vision. A signed informed consent was obtained from each participant before the experiment.

Attention network test. Figure 1 shows the schematic of the ANT. Stimuli consisted of a row of five horizontal black lines, with arrowheads pointing left or right, against a gray background (Fig. 1, two target conditions). A single arrow subtended $0.58^{\circ}$ of visual angle and the contours of adjacent arrows or lines were separated by $0.06^{\circ}$ of visual angle. The target was a leftward or rightward arrow at the center, flanked on either side by two arrows in the same direction (congruent condition), or in the opposite direction (incongruent condition). The stimuli (one center arrow and four flanker arrows) subtended a total $3.27^{\circ}$ of visual angle. The participants' task was to identify the direction of the target by pressing one key for the left direction and a second key for the right direction.

The row of five arrows was presented in one of two locations, either $1.06^{\circ}$ above or below the fixation cross. Thus, participants had to shift attention from the fixation point to the target location on each trial to determine the proper response. To measure the alerting and orienting benefits, a cue (an asterisk sign) was presented before the appearance of target. There were three cue conditions: no cue (baseline), center cue (at the fixation for alerting), and spatial cue (at the target location for alerting plus orienting). The operational definitions of the effects of the three attentional networks based on reaction time (RT) were as follows: alerting effect $=\mathrm{RT}_{\text {no cue }}-\mathrm{RT}_{\text {center cue }}$, orienting effect $=\mathrm{RT}_{\text {center cue }}-$ $\mathrm{RT}_{\text {spatial cue }}$, and conflict effect $=\mathrm{RT}_{\text {incongruent }}-\mathrm{RT}_{\text {congruent. }}$ The same contrasts were used for the calculation of these three effects based on accuracy. The contrasts for the alerting and orienting effects were flipped for the fMRI and ERP data analysis (i.e., alerting effect = center cue - no cue, orienting effect $=$ spatial cue - center cue). For the interpretations of the attentional network scores, see our previous publication (Fan and Posner, 2004).

Procedures. In each trial, depending on the condition, a cue was (center cue or spatial cue condition) or was not (no cue condition) presented for
$200 \mathrm{~ms}$. After a variable duration (300-1450 ms with an average of 550 $\mathrm{ms}$ ), the target (with flankers) was presented until a response was made. The time window for participants' response was $2000 \mathrm{~ms}$ after target onset. The duration between the onset of the target and the start of the next trial was also variable (3000-4200 ms with a mean of $3300 \mathrm{~ms})$. Each trial persisted $4050 \mathrm{~ms}$ on average. A fixation cross appeared in the center throughout the whole experiment.

Participants performed a total of six blocks of trials, each block lasting $\sim 8$ min. Each block consisted of 108 trials plus six buffer trials at beginning of each block. Trial order was counterbalanced as in the fMRI study (Fan et al., 2005). Participants were instructed to respond as quickly and accurately as possible with the left mouse button if the central arrow pointed left and the right mouse button if the central arrow pointed right using their left and right thumbs, respectively. The stimuli were the same as those used in the fMRI study except that the intervals between cue and target and between target and next trial were shorter. Stimulus presentation and behavioral response collection were performed using E-Prime (Psychology Software Tools, Pittsburgh, PA) on the experimental control personal computer, which was synchronized and communicated with the ERP data recording system. The trial events, including the cue and target type, and onset/offset time were sent to the ERP data recording system via a standard serial port.

ERP acquisition. Electrical data were recorded from 128 scalp sites using the Geodesic EEG Net Station (EGI, Eugene, OR) and the 128channel Geodesic Sensor Net (Tucker, 1993). The impedance of all electrodes except bad electrodes (no more than two per session) was $<40 \mathrm{k} \Omega$ at the beginning of the experiment. EEG was recorded using a $0.1-100 \mathrm{~Hz}$ bandpass filter. The signals were sampled at 250 samples/s and were digitized with a 12 bit analog-to-digital converter. All recordings were referenced to $\mathrm{Cz}$, then average-referenced off-line.

Behavioral data analysis. Trials with incorrect responses or with RTs longer than $1000 \mathrm{~ms}$ or shorter than $200 \mathrm{~ms}$ were excluded. The averaged RTs for each of the six combinations (three cue conditions by two target conditions) were calculated. The network scores were then calculated based on the operational definitions of the effects of the three attentional networks. The mean RTs from the two target conditions under each of the three cue conditions were averaged for the alerting and orienting calculation, and the mean RTs from the three cue conditions were averaged for the two target conditions separately for the calculation of the conflict effect. Grand averages of RT and accuracy, and the correlations among network scores were also calculated.

ERP data analysis. Initial off-line processing of the data was performed using Net Station software (version 2.0; EGI). ERPs were filtered using a $60 \mathrm{~Hz}$ notch filter before segmentation. ERPs for correct trials were segmented into epochs that were time-locked according to cue onset, target onset, or response. For the no cue trials, the waveforms during the no cue period were also segmented as if real cue events occurred. The average of these no cue segments, which represents the average overlap of adjacent events/trials, served as the baseline. The overlapping activity can be subtracted out using this baseline as demonstrated by other studies (Busse and Woldorff, 2003; Talsma and Woldorff, 2005). The time window was from $200 \mathrm{~ms}$ prestimulus to $800 \mathrm{~ms}$ poststimulus for both cue and target onset locked segments, and $400 \mathrm{~ms}$ before and $400 \mathrm{~ms}$ after response for response locked segments. Trials containing eye-blink, eye-movements, or an excessive number of bad channels $(>10)$ were identified using Net Station's moving window algorithm and excluded from additional analysis. Trials with incorrect responses were also excluded from additional analysis. In remaining trials, data for bad channels were replaced using spherical spline interpolation (Perrin et al., 1987). Segmented ERP data were then analyzed using Brain Electrical Source Analysis (BESA) 5.1 software (MEGIS Software, Grafelfing, Germany). First, epochs for analysis were defined both for the cue period ( $50 \mathrm{~ms}$ before cue onset to 450 $\mathrm{ms}$ after cue onset), the target period ( $100 \mathrm{~ms}$ before target onset to 700 $\mathrm{ms}$ after target onset), and the response-locked period (300 ms before response to $300 \mathrm{~ms}$ after response). Because EGI exports both "bad" and "good" segments, a second round of artifact rejection was performed using BESA. ERP data from one subject were excluded from the ERP and the subsequent analysis because artifacts during data recording.

Power spectrum analysis based on dipole modeling. The goal of the 
Table 1. The locations of the dipoles in the dipole source modeling

\begin{tabular}{lrrrr}
\hline & \multicolumn{4}{c}{ Talairach coordinates } \\
\cline { 2 - 5 } Region & BA & $x$ & \multicolumn{1}{c}{$y$} & \multicolumn{1}{c}{$z$} \\
\hline Alerting network & & & & \\
$\quad$ R superior temporal gyrus & 22 & 61 & -40 & 11 \\
L inferior parietal lobule & 40 & -50 & -20 & 21 \\
L fusiform gyrus & 37 & -42 & -62 & 0 \\
L inferior frontal gyrus & 47 & -32 & 27 & 0 \\
L superior parietal lobule & 7 & -36 & -46 & 50 \\
Orienting network & & & & \\
L fusiform gyrus & 37 & -34 & -60 & -5 \\
R fusiform gyrus & 37 & 30 & -47 & -6 \\
L precentral gyrus & 6 & -38 & -8 & 41 \\
R superior parietal lobule & 7 & 32 & -41 & 30 \\
L superior frontal gyrus & 6 & -10 & 7 & 57 \\
L superior parietal lobule & 7 & -28 & -72 & 28 \\
R postcentral gyrus & 2 & 57 & -21 & 43 \\
L precentral gyrus & 4 & -30 & -26 & 53 \\
Executive control network & & & & \\
L superior frontal gyrus & 6 & -16 & 4 & 44 \\
R inferior frontal gyrus & 45 & 36 & 26 & 15 \\
L fusiform gyrus & 37 & -36 & -60 & 1 \\
L inferior frontal gyrus & 47 & -34 & 20 & 5 \\
R middle frontal gyrus & 6 & 36 & -5 & 50 \\
R fusiform gyrus & 37 & 44 & -58 & 1 \\
R anterior cingulate gyrus & 32 & 6 & 36 & 26 \\
\hline
\end{tabular}

$B A$, Brodmann's area; $L$, left; $R$, right.

present analysis was to characterize the power changes associated with each attentional function across a wide range of frequencies. We first considered performing this analysis by analyzing power changes at the scalp level. However, the sensitivity of this method is decreased relative to analysis of power changes at the dipole level because all cortical sources contribute to the signal observed at any given electrode. We instead chose to model power changes at the dipole level for this and the following reasons. First, dipole-level analysis would allow us to characterize power changes associated with individual structures and, thus, makes it possible to associate power changes with the physical networks supporting each attentional function. Second, drawbacks associated with dipole modeling would be minimized because we were able to minimize experimenter bias and partially avoid the inverse problem by constraining dipole locations based on clusters of activation found in our previous fMRI study of the ANT (Fan et al., 2005). This was especially advantageous for the purposes of the current study because high-frequency components, such as those in the gamma band, may not be systematically colocalized with average evoked potentials (Tallon-Baudry et al., 2005); use of average evoked potentials to estimate dipole locations would present a challenge for the interpretation of changes in power of high-frequency components. Although average evoked potentials were used to estimate the direction of dipoles once they were placed, this method may still confer significant advantage over power-change analyses performed at the scalp level.

Dipole locations were based on clusters of activation identified in our previous fMRI study of the ANT (Fan et al., 2005). Table 1 shows the coordinates of the dipoles used in each analysis. For each condition, separate dipoles were located at the geometric center of mass of each cortically located region of activation as identified in the fMRI data. The relative level of activation for each voxel was not considered when calculating the center of mass. We followed the method described by (CrottazHerbette and Menon, 2006) to exclude brain regions such as the thalamus from dipole modeling that were activated in the fMRI data but that do not exhibit the columnar organization necessary to generate a field that can be recorded on the scalp. We also excluded the cerebellum from dipole modeling because the cerebellum generates its own independent electric activity that does not contribute to the scalp recording of the ERP (Tyner and Knott, 1989). Dipoles were positioned at the center of mass of all clusters identified in the fMRI study whose anatomical locations met the above criteria. Therefore, the alerting network had five dipole sources (the superior colliculus cluster, which had peaks in the left and right thalamus, and the cerebellar vermis cluster were excluded from the original seven clusters), the orienting network had eight dipole sources, and the executive control network had seven dipole sources (the thalamus and cerebellar vermis clusters were excluded from the original nine clusters). The dipole positions were fixed and the orientations were allowed to vary. Given that the ERP sample was a superset of the fMRI sample, the locations used were an approximation. We did not attempt to improve the fit by loosening the location constraint because this would introduce uncertainty. Empirical testing has shown that a small degree of deviation of a dipole's location does not significantly affect the model fitting.

The grand-averaged difference waveforms of the alerting, orienting, and conflict effects calculated from the cue-, target-, and response-locked ERPs of all participants were used for dipole direction estimation. The fitting periods for the cue-locked ERP were $0-450 \mathrm{~ms}$ after cue onset for alerting and orienting effects, $0-700 \mathrm{~ms}$ after target onset for conflict effect, and $300 \mathrm{~ms}$ preresponse to $300 \mathrm{~ms}$ postresponse for the responselocked analysis. To avoid fitting with bias, all dipoles were fitted simultaneously without any additional selection to link an ERP component to a specific dipole during a certain time period. Average surface points of the Geodesic Sensor Net provided by BESA were used. The BESA source model solutions for each network generated from the grand averaged ERPs were applied as montages to the individual participants' cue-, target-, or response-locked epochs of each trial, and power spectrum analyses were performed in BESA separately for each cue and target condition. This method can be defined as analysis of induced activity of frequency bands (Senkowski et al., 2005).

For the spectra, time-frequency sampling was $2 \mathrm{~Hz} / 25 \mathrm{~ms}$. BESA uses a complex demodulation to transform time-domain data into the timefrequency domain (Papp and Ktonas, 1977). This complex demodulation consists of a multiplication of the time-domain signal with a complex periodic exponential function, with a frequency equal to the frequency under analysis, and a subsequent low-pass filter. This low-pass filter is a finite impulse response filter of Gaussian shape in the timedomain, which is related to the envelope of the moving window in wavelet analysis. For our setting of a $2 \mathrm{~Hz} / 25 \mathrm{~ms}$ time-frequency sampling, this filter has a width in the frequency domain of $5.7 \mathrm{~Hz}$ and in the timedomain of $79 \mathrm{~ms}$ full width at half maximum. For more details about the BESA power spectrum analysis, see a previous publication (Hoechstetter et al., 2004). For sources of each attentional network, power spectra were computed for each trial and then were averaged for each participant under corresponding cue or target conditions.

Power changes compared with the average of prestimulus or postresponse baseline period were plotted. In the cue conditions (no cue, center cue, spatial cue), the time axis was from $50 \mathrm{~ms}$ precue to $450 \mathrm{~ms}$ postcue, the frequency axis was from 4 to $100 \mathrm{~Hz}$, and power change was defined as percentage of baseline (the average power of the $50 \mathrm{~ms}$ before stimulus onset). For the alerting montage, spectra were generated for all five sources in the no cue and center cue conditions, and the alerting effect was shown as the contrast of center cue minus no cue. For the orienting montage, spectra were generated for all eight sources in the center cue and spatial cue conditions, and the orienting effect was shown as the contrast of spatial cue minus center cue. In the target conditions (congruent and incongruent), the time axis was from $100 \mathrm{~ms}$ pretarget to 700 ms post-target, the frequency axis was from 4 to $100 \mathrm{~Hz}$, and power change was defined as percentage of baseline (the average power of the $100 \mathrm{~ms}$ before stimulus onset). For the response locked analysis, the time axis was from $300 \mathrm{~ms}$ preresponse to $300 \mathrm{~ms}$ postresponse, and the baseline was 200-300 ms postresponse. For both target-locked and responselocked executive control montages, spectra were generated for all seven dipole sources in the three cue (no cue, center cue, and spatial cue) by two target (incongruent and congruent) conditions, and the conflict effect was shown as the main effect of the difference between the power spectral changes under incongruent and congruent conditions. The modulation of the alerting (center) cue and the orienting (spatial) cue on target response was also examined.

Averaged power-change plots of each participant corresponding to each condition were filtered separately using a two-dimensional Gaussian kernel with an SD of $6 \mathrm{~Hz} / 75 \mathrm{~ms}$ before group analysis. This extra 
A

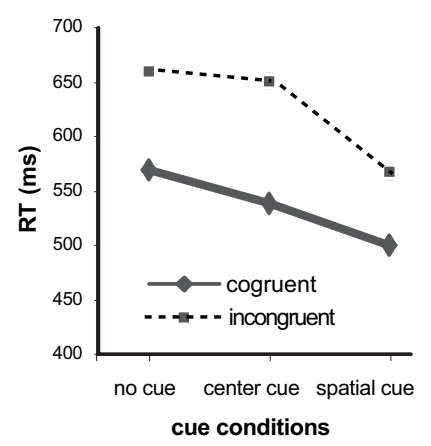

B

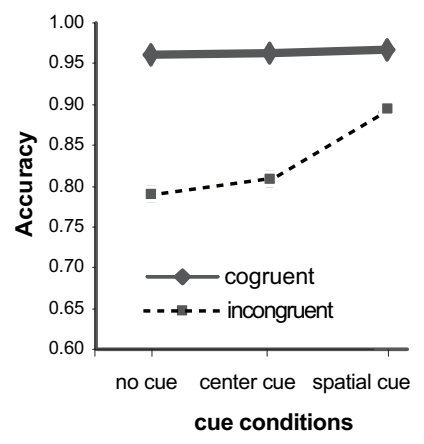

Figure 2. $\quad \boldsymbol{A}, \boldsymbol{B}$, The behavioral results of $\mathrm{RT}(\boldsymbol{A})$ and accuracy $(\boldsymbol{B})$ as factors of cue and target conditions.

step, in addition to using the moving window in the power spectrum analysis, increased the statistical power of group data analysis to detect differences between conditions at the cost of time and frequency resolution. This is similar to the use of spatial filers on raw or normalized blood oxygen level-dependent (BOLD) signal images to increase the power of statistical analysis for random effects in fMRI analysis. To determine the significance of an attentional effect, a paired $t$ test was performed on each data point in the two corresponding filtered power-change spectra. A final time-frequency plot was then generated with the color map representing the $t$ value of the power change difference between two conditions at each data point. This analysis was performed using Matlab 6.5 software (MathWorks, Natick, MA). Assuming an individual voxel type I error of $p<0.01$, a Monte Carlo simulation of the current study revealed that, to correct for multiple comparisons, the finding of significance could only be claimed if 20 contiguous original pixels, regardless of cluster shape, reached significance. For example, the finding of $|t(34)|>2.73$ for each pixel in a cluster of $5 \times 4$ pixels $(10 \mathrm{~Hz} \times 100 \mathrm{~ms})$ was needed for that cluster to reach significance.

Frequency bands were defined as theta $=4-8 \mathrm{~Hz}$, alpha $=8-14 \mathrm{~Hz}$, beta $=14-30 \mathrm{~Hz}$, and gamma $30-100 \mathrm{~Hz}$, with no overlap at the edge. The beta band includes the lower range beta $(14-18 \mathrm{~Hz})$ and the upper range beta $(18-30 \mathrm{~Hz}$ ) (Foffani et al., 2005; Merica and Fortune, 2005). The gamma-band $(30-100 \mathrm{~Hz})$ includes the low-gamma $(30-80 \mathrm{~Hz})$ and part of the high-gamma $(80-160 \mathrm{~Hz})$ range (Canolty et al., 2006). The ERP waveform for each dipole source was also averaged across all participants and plotted beside the power spectrum change map for comparison purposes.

\section{Results}

\section{Behavioral results}

Figure $2 A$ shows the RT results. The overall RT for all conditions was $580 \mathrm{~ms}$ (SD, $63 \mathrm{~ms}$ ). The RTs for each condition were $569 \mathrm{~ms}$ for no cue-congruent, $660 \mathrm{~ms}$ for no cue-incongruent, $538 \mathrm{~ms}$ for center cue-congruent, $650 \mathrm{~ms}$ for center cue-incongruent, 500 $\mathrm{ms}$ for spatial cue-congruent, and $568 \mathrm{~ms}$ for the spatial cueincongruent condition. The alerting, orienting, and conflict effects were $20 \mathrm{~ms}$ (SD, $16 \mathrm{~ms}$ ), $60 \mathrm{~ms}$ (SD, $25 \mathrm{~ms}$ ), and $90 \mathrm{~ms}$ (SD, $28 \mathrm{~ms}$ ), respectively. ANOVA showed that the factor of cue level was significant $\left(F_{(2,70)}=216.98 ; p<0.01\right)$, the factor of target condition (conflict effect, $536 \mathrm{~ms}$ for congruent vs $626 \mathrm{~ms}$ for incongruent $)$ was significant $\left(F_{(1,35)}=370.78 ; p<0.01\right)$, and the interaction between cue and target was significant, $F_{(2,70)}=$ 44.20; $p<0.01)$. The alerting by conflict and the orienting by conflict interactions were significant $\left(F_{(1,35)}=23.49\right.$ and $F_{(1,35)}=$ 88.60 ; $p$ values $<0.01$ ), indicating that the conflict was significantly greater in the center cue condition than in the other two conditions. Simple comparisons showed that the alerting and orienting effects were significant $\left(F_{(1,35)}=52.53\right.$ and $F_{(1,35)}=$
208.46; $p$ values $<0.01)$. The correlations between the alerting and orienting scores $(r=-0.08)$, between alerting and conflict $(r=-0.15)$, and between orienting and conflict $(r=0.12)$ were not significant ( $p$ values $>0.05$ ). The correlation between conflict and mean RT was significant $(r=0.49 ; p<0.01)$.

Figure $2 B$ shows the accuracy results. The overall accuracy was $90 \%$ (SD, 9\%). The accuracy for each condition was 96\% for no cue-congruent, $79 \%$ for no cue-incongruent, $96 \%$ for center cuecongruent, $81 \%$ for center cue-incongruent, $97 \%$ for spatial cuecongruent, and $89 \%$ for spatial cue-incongruent. The alerting, orienting, and conflict effects were $-1 \%(\mathrm{SD}, 4 \%),-4 \%$ (SD, $4 \%$ ), and $13 \%(\mathrm{SD}, 7 \%)$, respectively. ANOVA showed that the factor of cue level was significant $\left(F_{(2,70)}=37.18\right.$; $\left.p<0.01\right)$, the factor of target condition (conflict effect) was significant $\left(F_{(1,35)}\right.$ $=117.09 ; p<0.01)$, and the interaction between cue and target was significant $\left(F_{(2,70)}=29.82 ; p<0.01\right)$. The alerting by conflict interaction was not significant $\left(F_{(1,35)}=1.84 ; p>0.05\right)$; however, the orienting by conflict interaction was significant $\left(F_{(1,35)}=\right.$ 35.04; $p<0.01$ ), indicating that the conflict was significantly less in the spatial cue than in the center cue condition. Simple comparisons showed that the alerting effect was not significant $\left(F_{(1,35)}\right.$ $=3.36$; $p=0.08$ ), but orienting and conflict effects were significant $\left(F_{(1,35)}=36.24\right.$ and $F_{(1,35)}=119.07 ; p$ values $\left.<0.01\right)$. The correlation between the alerting and orienting scores $(r=-0.47)$ was significant ( $p<0.01$ ), possibly because the center cue condition was used in the calculation of both alerting and orienting scores. The correlation between alerting and conflict $(r=-0.13)$ was not significant $(p>0.05)$, although the correlation between the orienting and conflict $(r=0.69)$ was significant $(p<0.01)$.

\section{Topographical maps and ERP waveforms}

Figure 3 shows the topographical map of the voltage distribution at 350, 225, 450, and $100 \mathrm{~ms}$ for alerting, orienting, and both target- and response-locked executive control effects, respectively, as difference waveforms. These time points were selected based on the latencies of the ERP peaks and maxima of power change for each network. To enhance the statistical power, the average waveforms of the E68 and surrounding electrodes (E60, E61, E62, E67, E73, E78, E79, E86) for alerting, E59 and surrounding electrodes (E51, E52, E58, E60, E65) for orienting, and E6 and surrounding electrodes (E5, E7, E12, E13, E107, E113) for target-locked and response-locked ERPs were averaged and compared between contrasting conditions in the averaged data of individual participants. The E68 and E59 electrodes were at the maxima of the topographical maps for alerting and orienting effects, respectively. The E6 and surrounding electrodes are from the medial-frontal channels, which have been shown to relate to error-related negativity (ERN) and theta-band ERP (Luu et al., 2004). The $p$ values for the $t$ tests of the difference between two conditions were also shown for the periods of 300-400 ms for alerting, 175-275 ms for orienting, 500-600 ms for targetlocked, and 0-200 for response-locked ERPs of executive control. The N2 component can be seen from the cue- and targetlocked, but not the response-locked responses.

\section{Time-frequency properties of attentional networks}

The dipole sources for alerting, orienting, and executive control target- and response-locked effects explained 86, 91, 92, and 83\% variance, respectively, for the modeled periods of the grand average differential ERPs.

\section{Alerting network}

Figure 4 shows that alerting evoked changes in rhythmic power of the alerting network. The most noticeable finding is the decrease 

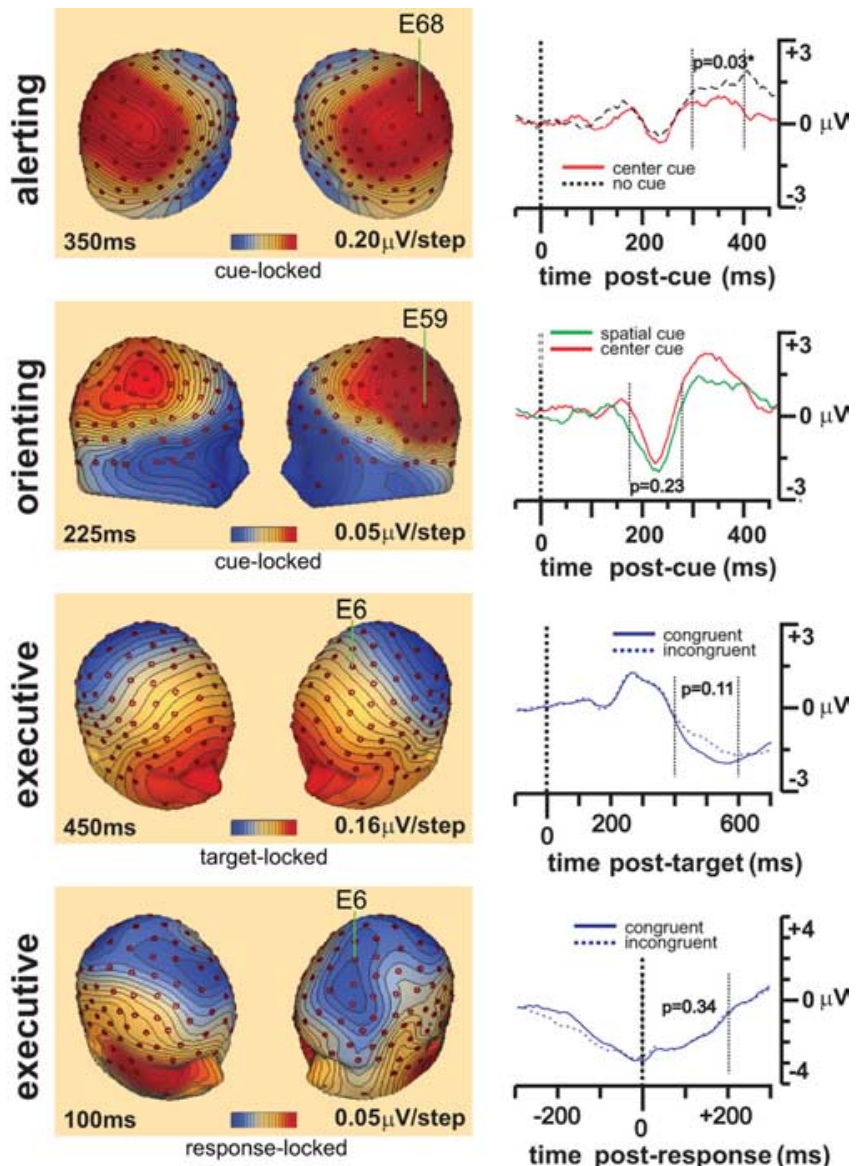

Figure 3. The voltage scalp distribution maps for alerting at $350 \mathrm{~ms}$, orienting at $225 \mathrm{~ms}$, and both target- and response-locked conflict effects at 450 and $100 \mathrm{~ms}$ respectively. Left, Voltage scalp distribution maps; right, stimulus-locked waveform from averaged channels centered at E68 for alerting, E59 for orienting, and E6 for target- and response-locked executive control effects are displayed for comparison. in theta-, alpha-, and beta-band $(4-30 \mathrm{~Hz}$, centered at alphaband) power during 200-450 ms after cue, which is seen throughout the entire alerting network except in the left inferior frontal gyrus (Fig. 4, center cue column). There was an increase in theta activity in the right superior temporal gyrus and left inferior frontal gyrus (Fig. 4, alerting effect column). The decrease of gamma-band activity found in the right superior temporal gyrus and other dipole sources comparing the center cue with no cue condition (Fig. 4, alerting effect column) may be caused by both a significant gamma-band activity decrease under the center cue condition (as we can see from the center cue column of Fig. 4, there is no significant gamma band activity decrease) and a gamma-band activity increase under the no cue condition related to overlap effects generated by other events. Grand-averaged source waveforms of the alerting network are provided for comparison; notice that each source has a unique waveform profile. Importantly, if one were to examine the ERPs exclusively, the cohesive nature of the theta-, alpha-, and beta-band power decrease within the alerting network would be obscured.

\section{Orienting network}

Figure 5 shows the time-frequency pattern of the orienting network. There was a specific increase in gamma-band power $(30-80 \mathrm{~Hz})$ with some beta-band components $\sim 200 \mathrm{~ms}$ after spatial cue onset (Fig. 5, spatial cue column). This specific increase in gamma-band power was not elicited by the center cue condition (Fig. 5, center cue column). For the spatial cue minus center cue contrast, the gamma-band power increase effect was most pronounced in the fusiform gyrus bilaterally, the right superior parietal lobule near the anterior intraparietal sulcus, the right postcentral gyrus, and the second region of the left precentral gyrus (BA4) (Fig. 5, orienting effect column). The left superior frontal gyrus dipole source showed a different pattern with more $60-80 \mathrm{~Hz}$ of gamma-band and low-frequency power $(<30$ $\mathrm{Hz}$ ) activity increase. The second left precentral gyrus source (BA4) also showed a low-frequency power $(<30 \mathrm{~Hz})$ increase. The left precentral gyrus and left superior parietal lobule did not show significant gamma-band power change. Again, source waveforms from each source are presented for comparison. Although each source has a unique source waveform profile, all of them appeared to be very similar between the two cue types.

\section{Executive control network}

Examination of the time-frequency pattern of the executive control network in Figure 6 yielded several interesting findings. First, onset of targets with either incongruent or congruent flankers under no cue or center cue conditions evoked a strong increase in gamma-band (30-90 $\mathrm{Hz}$ ) power together with some frequency components of beta-band during the $100-300$ ms period after target onset, similar to the power change related to spatial cue in Figure 5. This effect was most pronounced in the frontal dipole sources of the left superior frontal gyrus, the right inferior frontal gyrus, and the left inferior frontal gyrus, and was also seen clearly in the left fusiform gyrus (Fig. 6, no cue and center cue columns). When the participant was oriented by the spatial cue before the
Figure 4. Time-frequency pattern of the alerting network. The source column shows the dipole sources used for the dipole modeling of the alerting network. The center cue and no cue columns are the power change (percentage) after onset of center cue and no cue compared with baseline. The alerting effect column represents the alerting effect as the difference of the center cue column minus the no cue column shown with $t$ maps. 
onset of the target, the gamma-band activity was highly attenuated (Fig. 6, spatial cue columns). This may indicate that the gammaband power increase found in these dipole sources during the target response in the executive control network is related to the orienting processing needed under no cue and center cue conditions after the target and flanker appears and is not specifically related to executive control.

Second, for both target types there was a long-latency decrease in the power of frequencies $<30 \mathrm{~Hz}$ centered at alphaband throughout most sources of the network. This is very similar to that as seen after presentation of center and spatial cues (Figs. 4, 5). The attenuated theta-, alpha-, and beta-band $(<30 \mathrm{~Hz})$ power change for the target response under center cue and spatial cue conditions compared with the no cue condition may indicate that the decrease in power of these bands found in Figure 6 is attributable mainly to the alerting function recruited for the target response when no cue proceeded the target.

Finally, and most importantly, the main effect of conflict (incongruent minus congruent, the conflict effect column) showed a complex pattern. There were source-specific increases in gamma-band activity seen at early latency in some frontal dipole sources (right inferior frontal gyrus, right middle frontal gyrus, and right ACC; $<400 \mathrm{~ms}$ ), beta- and low gammaband $(30-60 \mathrm{~Hz})$ power decreases during 400-600 ms (which were close to the average RT of $580 \mathrm{~ms}$ and were most significant in right the inferior frontal gyrus, right middle frontal gyrus, and right fusiform gyrus), decreases in beta-band power at late latency in the right middle frontal gyrus, and even lower bands of theta and alpha in other regions such as the left fusiform gyrus during 500-700 ms. For the right inferior frontal gyrus, there was also an increase in theta- and alpha-band power before the response. The left inferior frontal gyrus source showed a more complex pattern with an increase in late-latency low $(<20 \mathrm{~Hz})$ and high $(>80 \mathrm{~Hz})$ band power.

To the right of Figure 6, source waveforms from each source are presented for comparison. There were small (the N2 component for the early latency) and nonsignificant differences (the P300 for the later 400-600 ms component) between congruent and incongruent target conditions.

We also found theta- and alpha-band power bursts after the button response in the right fusiform gyrus for the spatial cuecongruent target condition, which had an average RT of $500 \mathrm{~ms}$. The complex patterns of the executive control effect from the dipole sources of the executive control network may also relate to the shifting of frequency components along the time axis under the incongruent condition for conflict processing. Based on these findings, we decided to investigate further by analyzing the response-locked power spectral changes. Figure 7 shows the complex pattern of results obtained from the response-locked analysis for the executive control network. Similar to the late power decreases found for the target-locked analysis (Fig. 6), there were

\section{Orienting Network}

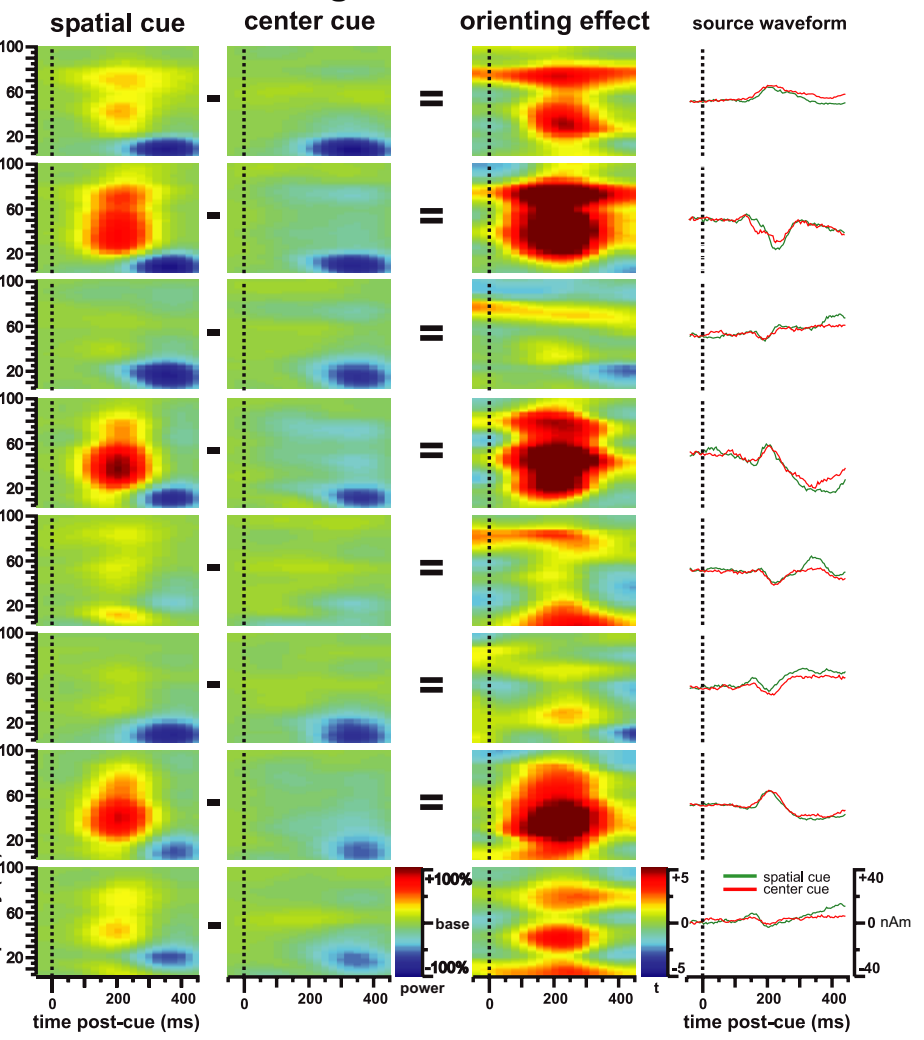

Figure 5. Time-frequency pattern of the orienting network. The source column shows the dipole sources used for the dipole spatial cue and center cue compared with baseline. The orienting-effect column represents orienting effect as the difference of the spatial cue column minus the center cue column shown with $t$ maps.

broad-band power decreases before the responses made to targets accompanied by incongruent flankers compared with those with congruent flankers (Fig. 7, conflict resolution column). There was also a gamma-band power increase in almost all sources typically after response. Consistent with the target-locked analysis, the theta burst after target response can be seen clearly from the right fusiform gyrus dipole source. The ACC and left superior frontal gyrus dipole sources showed the beta-band burst and increased gamma power after response.

In summary, alerting was most accompanied by a decrease in theta-, alpha-, and beta-band activity after a warning signal (200$450 \mathrm{~ms}$ ). Orienting was associated with an increase in gammaband activity either after a cue $(\sim 200 \mathrm{~ms})$ indicating the target location or when a target requires a shift of attention to the target location. For the target-locked analysis, the conflict processing in the executive network involved an early $(<400 \mathrm{~ms})$ increase of a broad-band activity including gamma-band and a late $(>400 \mathrm{~ms})$ decrease of a broad-band activity including theta-, alpha-, and low gamma-bands. For the response-locked analysis of conflict resolution, the decrease of broad-band activity, including theta-, alpha-, and low gamma- before response and theta-, beta-, and gammaband bursts near or after response, were also observed.

\section{Discussion}

Each of the three attentional networks appears to have a distinct pattern of synchronized frequency change. In the alerting network, the decrease in theta-, alpha-, and beta-band power corresponds to the usual desynchronization of electrical activity after a warning. One interpretation of this finding could be that the 


\section{Executive Control Network - target locked}

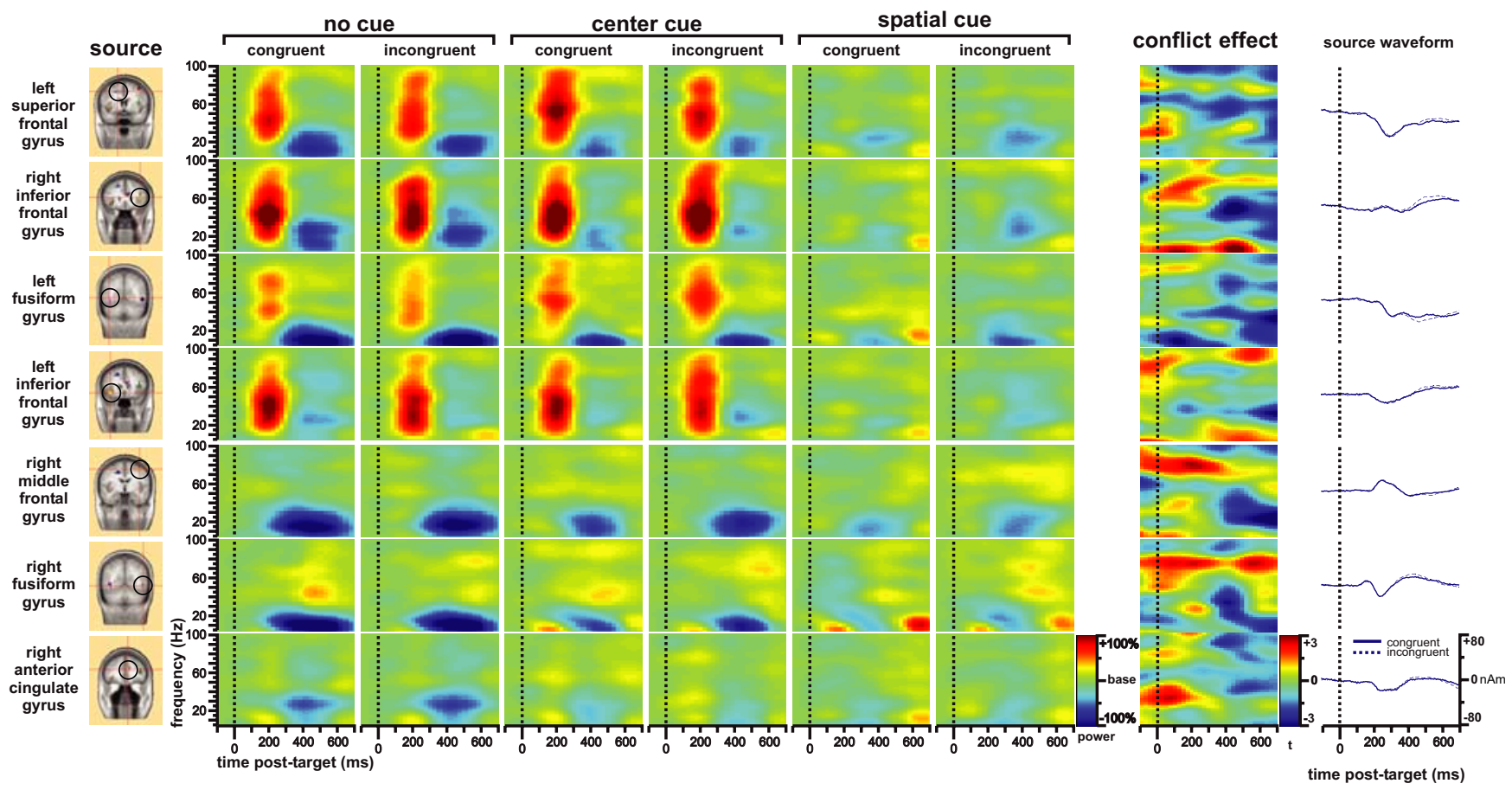

Figure 6. Time-frequency pattern of target-locked analysis of the executive control network. The source column shows the dipole sources used for the dipole modeling of the target-locked ERP. The next six columns are the power change (percentage) after onset of target with congruent and incongruent flankers compared with baseline under the no cue, center cue, and spatial cue conditions, respectively. The conflict-effect column represents the main effect of conflict (incongruent minus congruent conditions) as $t$ maps.

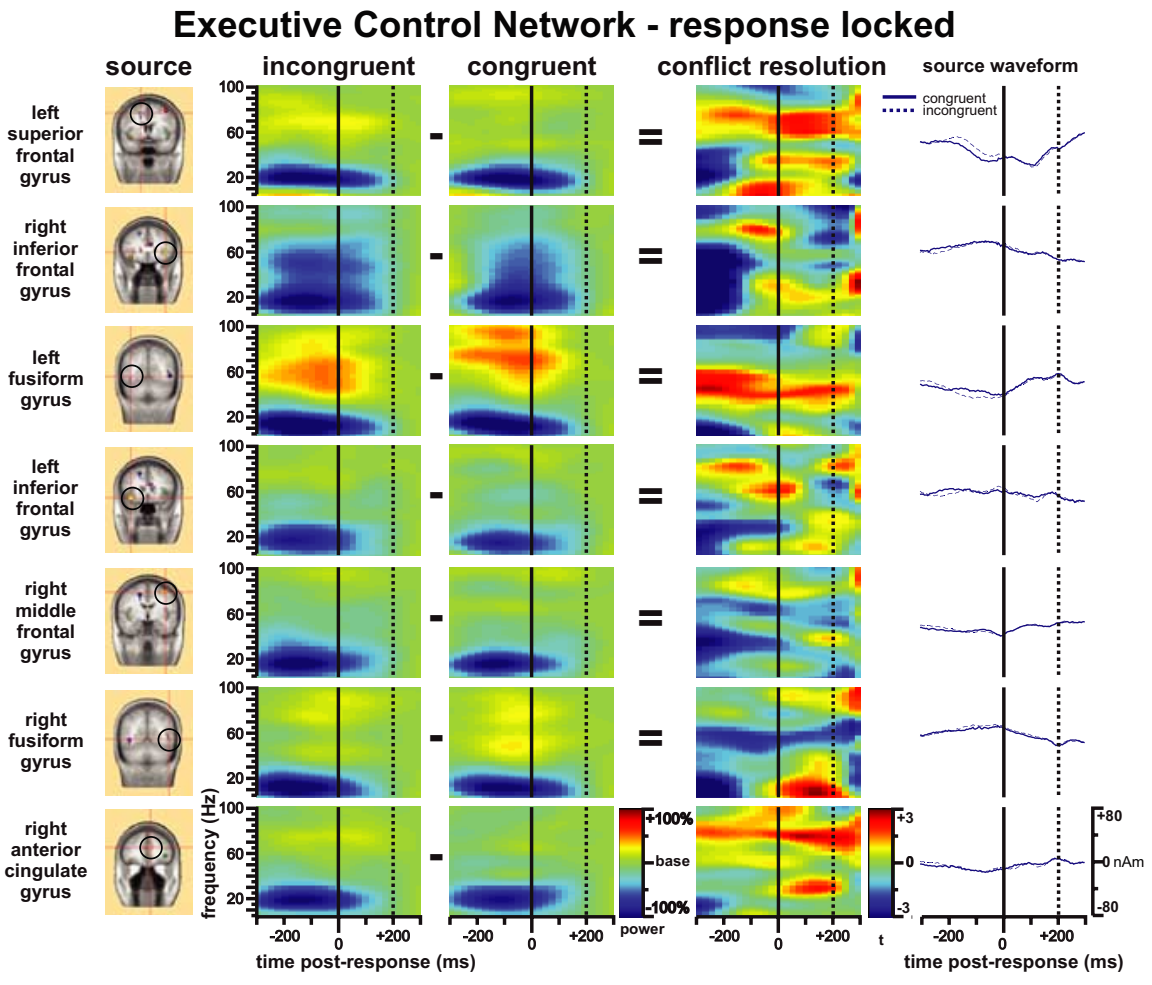

Figure 7. Time-frequency pattern of response-locked analysis of the executive control network. The source column shows the dipole sources used for dipole modeling of the response-locked ERP. The incongruent and congruent columns are for the power change (percentage) before and after response with incongruent and congruent flankers compared with the baseline. The conflictresolution column represents the response-locked conflict effect as incongruent minus congruent conditions shown as $t$ maps. desynchronization occurs in response to any visual stimulus, and is not specific to alerting. However, the abrupt onset of any visual stimulus is known to attract attention (Yantis and Jonides, 1984), therefore we may also interpret the standard alpha suppression effect to be related to attention. In this study, the reduction of activity was not just in the alpha band, but was much more broadly distributed in the frequency domain. The fact that cued target responses showed attenuated theta-, alpha-, and beta-band power reduction may provide support for the relationship between these frequency domains and the alerting function. The across-dipole desynchronization of theta-, alpha-, and beta-bands found in this study may indicate the involvement of thalamocortical circuits, including norepinephrine systems involved in alerting (Marrocco and Davidson, 1998). The norepinephrine system has been shown to be involved in general preparatory states. In our study, the decrease in theta-, alpha-, and beta-band power occurs $\sim 200 \mathrm{~ms}$ after the warning in all of the cued conditions. This result indicates a mechanism such as alerting resulting from information that a target will occur, and suggests a relationship between these frequency bands and the alerting function. In addition, it may be useful to consider the distinction between sources 
and sites of the alerting network. The fusiform activity may be the site of origin and not part of the alerting network per se, and may be related to the fact that we just presented a stimulus in the visual modality or modulated by the sources of the alerting network (e.g., the thalamus).

Evidence from cellular studies in monkeys indicates that attended targets induce changes in gamma-band activity in the visual cortex (Fries et al., 2001; Womelsdorf et al., 2006). Previous ERP studies have shown enhancement of the P1 and N1 components of the ERP in areas of extrastriate visual cortex (Hillyard et al., 2004). It has been argued that these visual effects involve orienting of attention and are orchestrated from the parietal and frontal cortices (Corbetta and Shulman, 2002; Hillyard et al., 2004). Our study clearly shows gamma activity $\sim 200 \mathrm{~ms}$ after a visual cue that indicated the presentation of a target, even before the target was actually presented. However, if no cue was present, gamma-band enhancement occurred $\sim 200 \mathrm{~ms}$ after the target. The relative enhanced gamma oscillation in the fusiform gyrus, right superior parietal lobule, and some other regions related to orienting found in the present study is consistent with the study of attentional modulation of gamma-band oscillation found previously in the fusiform gyrus (Tallon-Baudry et al., 2005) and for attention to a moving stimulus (Gruber et al., 1999). Together, these results and the present study suggest that gamma frequency enhancement arises as a result of the function of the orienting network.

The executive control network showed an early increase in power in a broad range of frequencies, including gamma-band, after the presentation of targets with incongruent flankers compared with the presentation of targets with congruent flankers. This is consistent with previous studies on the time course of the electrical activity of the ACC (Snyder et al., 1995; Abdullaev and Posner, 1997). The early latency gamma-band power increase in medial-frontal channels has been found to be related to the effect of attention on the integrative process (Herrmann et al., 1999; Senkowski et al., 2005). The same phenomena observed in the ACC in the present study suggests that the ACC is involved in such an integrative process. The ACC and lateral prefrontal cortex are integral parts of the executive attention network. Many studies indicate that the ACC plays a major role involving response selection and monitoring (Botvinick et al., 2001; Fan et al., 2003). In a study investigating the involvement of the ACC in action monitoring, the N2 component and ERN were modeled with a dipole located in the caudal ACC, with two additional dipoles in the left prefrontal region and the left superior parietal lobule to explain the later error positivity $P_{\mathrm{E}}$ (Van Veen and Carter, 2002). An EEG-fMRI combined study showed that ERN to false alarms relates to $\mathrm{MRI}$ activation of caudal and rostral ACC, whereas N2 to correct rejections (conflict monitoring) correlated with $\mathrm{AMRI}$ activation of caudal ACC and dorsal lateral prefrontal cortex (Mathalon et al., 2003).

The time course of the preresponse theta-, beta-, and low gamma-band activity decrease and the postresponse theta-, beta-, and gamma-band bursts is also consistent with the time course of frontal midline theta bursts after correct speeded response (Makeig et al., 2004), and after the ERN (Luu and Tucker, 2001; Luu et al., 2004). It has been suggested that postresponse coherent theta activity is associated with cognitive function (Caplan et al., 2003) and might enhance the speed, salience, and reliability of spike-based communication between brain regions for context updating (Makeig et al., 2004). Microelectrode recording has provided direct evidence for the task-related theta generation
(Wang et al., 2005). It has also been suggested that changes in alpha- and lower beta-band activity $(8-20 \mathrm{~Hz})$ reflect specific attentional demands (Ray and Cole, 1985). The increase in thetaband power after the response may also reflect the potential contribution of theta oscillations to long-range communications for cognitive processing by phase-locking to high gamma power (Canolty et al., 2006). In this context, the increase in theta oscillations may be critical for postresponse cognitive processing and context updating between brain regions.

Although the three attentional networks are separable, they do interact to optimize attentional performance. First, alerting improves the speed of target response (Fan et al., 2007). It has been reported that there is a P300 amplitude enhancement if the cue predicts the cue-target interval (Miniussi et al., 1999). Alerting involves enhancement of the N2 component followed by overlapping modulation of the $\mathrm{P} 300$ and contingent negative variation components (Nobre, 2001; Griffin et al., 2002). The enhanced N2 component for $100 \%$ predictive novel stimulus facilitates target detection, whereas a novel stimuli with no predictive value generates increased novelty $\mathrm{P} 3 \mathrm{a}$ and target $\mathrm{P} 3 \mathrm{~b}$ responses (Suwazono et al., 2000). The gamma-band power increase and theta-, alpha-, and beta-band power decreases have a similar time course to the N2 and the P300 components. Second, the orienting function can reduce the conflict effect by focusing attention on the target to minimize the conflict generated by flankers or by reducing the resource requirement for orienting during the target response after the orienting cue. The gammaband activity for the target response after a spatial cue was dramatically attenuated. Therefore, the more efficient conflict processing for the spatial cue condition may relate to the reduction in need for gamma activity to perform orienting for the target response and conflict processing after a spatial cue.

In a magnetoencephalography study of effects of spatialselective attention on oscillatory neuronal dynamics, the suppression of alpha, reduction of beta, and enhanced gamma-band oscillations were mapped onto the parieto-occipital, sensorimotor, and somatosensory cortices (Bauer et al., 2006). The relationship between fMRI BOLD signal and frequency components supports the use of frequency analysis of ERP and EEG data to examine the temporal mechanisms of neural networks. A study of the relationship between the BOLD signal and underlying neuronal activity in the human auditory cortex showed a negative correlation between the BOLD signal and local field potentials in the low-frequency band $(<30 \mathrm{~Hz})$, but a positive correlation with the high-frequency band $(>30 \mathrm{~Hz})$ activity (Mukamel et al., 2005). If these finding are applied to our current data, they suggest that the altered frequency operations that accompany orienting, alerting, and conflict processing would all be expected to produce increases in the BOLD signal. This expectation fits well with the results of alerting and orienting network, except that conflict processing involves a more complex pattern.

The present study demonstrates the importance of examining power spectra in addition to ERPs to better understand the temporal characteristics of each attentional network. In some cases, the frequency band changes offer information that is not available from ERP components alone. For example, it has been shown that high-frequency components (e.g., gamma oscillation) do not contribute to the averaged evoked potentials (Liang et al., 2005) and that gamma oscillation and evoked potentials are not systematically colocalized (Tallon-Baudry et al., 2005). We have circumvented this issue by using fMRI results to constrain dipole locations and by calculating average power changes from 
individual trials, rather than calculating power changes from averaged ERPs. Finally, the finding of characteristic time-frequency patterns for each attentional function along with evidence of a distinct anatomy (Fan et al., 2005) supports the separation of attentional functions into distinct networks and the association of specific attentional networks with specific oscillation patterns, although the networks clearly interact in the performance of many acts of attention.

\section{References}

Abdullaev YG, Posner MI (1997) Time course of activating brain areas in generating verbal associations. Psychol Sci 8:56-59.

Babiloni C, Miniussi C, Babiloni F, Carducci F, Cincotti F, Del Percio C, Sirello G, Fracassi C, Nobre AC, Rossini PM (2004) Sub-second "temporal attention" modulates alpha rhythms. A high-resolution EEG study. Brain Res Cogn Brain Res 19:259-268.

Bauer M, Oostenveld R, Peeters M, Fries P (2006) Tactile spatial attention enhances gamma-band activity in somatosensory cortex and reduces lowfrequency activity in parieto-occipital areas. J Neurosci 26:490-501.

Botvinick MM, Braver TS, Barch DM, Carter CS, Cohen JD (2001) Conflict monitoring and cognitive control. Psychological Rev 108:624-652.

Busse L, Woldorff MG (2003) The ERP omitted stimulus response to "nostim" events and its implications for fast-rate event-related fMRI designs. NeuroImage 18:856-864.

Canolty RT, Edwards E, Dalal SS, Soltani M, Nagarajan SS, Kirsch HE, Berger MS, Barbaro NM, Knight RT (2006) High gamma power is phaselocked to theta oscillations in human neocortex. Science 313:1626-1628.

Caplan JB, Madsen JR, Schulze-Bonhage A, Aschenbrenner-Scheibe R, Newman EL, Kahana MJ (2003) Human theta oscillations related to sensorimotor integration and spatial learning. J Neurosci 23:4726-4736.

Corbetta M, Shulman GL (2002) Control of goal-directed and stimulusdriven attention in the brain. Nat Rev Neurosci 3:201-215.

Crick F, Koch C (1990) Some reflections on visual awareness. Cold Spring Harb Symp Quant Biol 55:953-962.

Crottaz-Herbette S, Menon V (2006) Where and when the anterior cingulate cortex modulates attentional response: combined fMRI and ERP evidence. J Cogn Neurosci 18:766-780.

Fan J, Posner M (2004) Human attentional networks. Psychiatr Prax 31 [Suppl 2]:210-214.

Fan J, McCandliss BD, Sommer T, Raz A, Posner MI (2002) Testing the efficiency and independence of attentional networks. J Cogn Neurosci 14:340-347.

Fan J, Flombaum JI, McCandliss BD, Thomas KM, Posner MI (2003) Cognitive and brain consequences of conflict. NeuroImage 18:42-57.

Fan J, McCandliss BD, Fossella J, Flombaum JI, Posner MI (2005) The activation of attentional networks. NeuroImage 26:471-479.

Fan J, Kolster R, Ghajar J, Suh M, Knight RT, Sarkar R, McCandliss BD (2007) Response anticipation and response conflict: an event-related potential and functional magnetic resonance imaging study. J Neurosci 27:2272-2282.

Foffani G, Bianchi AM, Baselli G, Priori A (2005) Movement-related frequency modulation of beta oscillatory activity in the human subthalamic nucleus. J Physiol (Lond) 568:699-711.

Fries P, Reynolds JH, Rorie AE, Desimone R (2001) Modulation of oscillatory neuronal synchronization by selective visual attention. Science 291:1560-1563.

Griffin IC, Miniussi C, Nobre AC (2002) Multiple mechanisms of selective attention: differential modulation of stimulus processing by attention to space or time. Neuropsychologia 40:2325-2340.

Gruber T, Muller MM, Keil A, Elbert T (1999) Selective visual-spatial attention alters induced gamma band responses in the human EEG. Clin Neurophysiol 110:2074-2085.

Herrmann CS, Mecklinger A, Pfeifer E (1999) Gamma responses and ERPs in a visual classification task. Clin Neurophysiol 110:636-642.

Herrmann CS, Munk MH, Engel AK (2004) Cognitive functions of gamma-band activity: memory match and utilization. Trends Cogn Sci 8:347-355.
Hillyard SA, Di Russo F, Martinez A (2004) The imaging of visual attention. In: Functional neuroimaging of visual cognition attention and performance XX (Kanwisher N, Duncan J, eds), pp 381-390. Oxford: Oxford UP.

Hoechstetter K, Bornfleth H, Weckesser D, Ille N, Berg P, Scherg M (2004) BESA source coherence: a new method to study cortical oscillatory coupling. Brain Topogr 16:233-238.

Kahana MJ (2006) The cognitive correlates of human brain oscillations. J Neurosci 26:1669-1672.

Kahana MJ, Seelig D, Madsen JR (2001) Theta returns. Curr Opin Neurobiol 11:739-744.

Keil A, Gruber T, Muller MM (2001) Functional correlates of macroscopic high-frequency brain activity in the human visual system. Neurosci Biobehav Rev 25:527-534.

Liang H, Bressler SL, Buffalo EA, Desimone R, Fries P (2005) Empirical mode decomposition of field potentials from macaque $\mathrm{V} 4$ in visual spatial attention. Biol Cybern 92:380-392.

Luu P, Tucker DM (2001) Regulating action: alternating activation of midline frontal and motor cortical networks. Clin Neurophysiol 112:1295-1306.

Luu P, Tucker DM, Makeig S (2004) Frontal midline theta and the errorrelated negativity: neurophysiological mechanisms of action regulation. Clin Neurophysiol 115:1821-1835.

Makeig S, Delorme A, Westerfield M, Jung TP, Townsend J, Courchesne E, Sejnowski TJ (2004) Electroencephalographic brain dynamics following manually responded visual targets. PLoS Biol 2:e176.

Marrocco RT, Davidson MC (1998) Neurochemistry of attention. In: The attentive brain (Parasuraman R, ed), pp 35-50. Cambridge, MA: MIT.

Mathalon DH, Whitfield SL, Ford JM (2003) Anatomy of an error: ERP and fMRI. Biol Psychol 64:119-141.

Merica H, Fortune RD (2005) Spectral power time-courses of human sleep EEG reveal a striking discontinuity at approximately $18 \mathrm{~Hz}$ marking the division between NREM-specific and wake/REM-specific fast frequency activity. Cereb Cortex 15:877-884.

Miniussi C, Wilding EL, Coull JT, Nobre AC (1999) Orienting attention in time. Modulation of brain potentials. Brain 122:1507-1518.

Mukamel R, Gelbard H, Arieli A, Hasson U, Fried I, Malach R (2005) Coupling between neuronal firing, field potentials, and FMRI in human auditory cortex. Science 309:951-954.

Nikolaev AR, Ivanitsky GA, Ivanitsky AM, Posner MI, Abdullaev YG (2001) Correlation of brain rhythms between frontal and left temporal (Wernicke's) cortical areas during verbal thinking. Neurosci Lett 298:107-110

Nobre AC (2001) Orienting attention to instants in time. Neuropsychologia 39:1317-1328.

Papp N, Ktonas P (1977) Critical evaluation of complex demodulation techniques for the quantification of bioelectrical activity. Biomed Sci Instrum 13:135-145.

Perrin F, Pernier J, Bertrand O, Giard MH, Echallier JF (1987) Mapping of scalp potentials by surface spline interpolation. Electroencephalogr Clin Neurophysiol 66:75-81.

Posner MI, Petersen SE (1990) The attention system of the human brain. Annu Rev Neurosci 13:25-42.

Ray WJ, Cole HW (1985) EEG activity during cognitive processing: influence of attentional factors. Int J Psychophysiol 3:43-48.

Senkowski D, Talsma D, Herrmann CS, Woldorff MG (2005) Multisensory processing and oscillatory gamma responses: effects of spatial selective attention. Exp Brain Res 166:411-426.

Snyder AZ, Abdullaev YG, Posner MI, Raichle ME (1995) Scalp electrical potentials reflect regional cerebral blood flow responses during processing of written words. Proc Natl Acad Sci USA 92:16891693.

Suwazono S, Machado L, Knight RT (2000) Predictive value of novel stimuli modifies visual event-related potentials and behavior. Clin Neurophysiol 111:29-39.

Tallon-Baudry C, Bertrand O, Henaff MA, Isnard J, Fischer C (2005) Attention modulates gamma-band oscillations differently in the human lateral occipital cortex and fusiform gyrus. Cereb Cortex $15: 654-662$. 
Talsma D, Woldorff MG (2005) Selective attention and multisensory integration: multiple phases of effects on the evoked brain activity. J Cogn Neurosci 17:1098-1114.

Tucker DM (1993) Spatial sampling of head electrical fields: the geodesic sensor net. Electroencephalogr Clin Neurophysiol 87:154-163.

Tyner FS, Knott JR (1989) Fundamentals of EEG technology, Vol II, Clinical correlates. New York: Raven.

Van Veen V, Carter CS (2002) The timing of action-monitoring processes in the anterior cingulate cortex. J Cogn Neurosci 14:593-602.

Wang C, Ulbert I, Schomer DL, Marinkovic K, Halgren E (2005) Responses of human anterior cingulate cortex microdomains to error detection, conflict monitoring, stimulus-response mapping, familiarity, and orienting. J Neurosci 25:604-613.
Womelsdorf T, Fries P, Mitra PP, Desimone R (2006) Gamma-band synchronization in visual cortex predicts speed of change detection. Nature 439:733-736.

Worden MS, Foxe JJ, Wang N, Simpson GV (2000) Anticipatory biasing of visuospatial attention indexed by retinotopically specific alphaband electroencephalography increases over occipital cortex. J Neurosci 20:RC63.

Yamagishi N, Goda N, Callan DE, Anderson SJ, Kawato M (2005) Attentional shifts towards an expected visual target alter the level of alpha-band oscillatory activity in the human calcarine cortex. Brain Res Cogn Brain Res 25:799-809.

Yantis S, Jonides J (1984) Abrupt visual onsets and selective attention: evidence from visual search. J Exp Psychol Hum Percept Perform 10:601-621. 